1

2

3

4

5

6

7

8

9

10

11

12

13

14

15

16

17

18

19

20

21

22

23

24

25

26

27

28

29

30

31

32

33

34

35

36
This is a non-peer reviewed preprint submitted to EarthArXiv. The article is under review in International Journal of Remote Sensing. 


\section{Machine Learning on Greenest Pixels for Crop Mapping}

zsun@gmu.edu; ldi@gmu.edu (corresponding author); hfang1288@gmail.com

Center for Spatial Information Science and Systems

George Mason University

4087 University Dr STE 3100

44

Abstract: Remotely sensed signals from crop fields are full of variabilities due to the complex interactions among the environment, seeds, climate, market, and farmers. It is a common phenomenon that the crops in neighbouring fields are in different growing stages, e.g., the corns are in the V5 leaf stage in one field and V10 stage in another neighbouring field. The phenomenon results in remote sensing images that are nonideal for producing crop maps as the crops are in different phenological stages across the fields. For instance, July is the best month for monitoring soybeans but bad for monitoring winter wheat which has been harvested before July. Most of the wheat fields are already harvested in July and appear like fallow fields on satellite images. This paper proposes a customized classification approach based on greenest pixels (GP) to enhance the quality of the satellite images for mapping. The greenest pixels are obtained by calculating the highest NDVI (Normalized Difference Vegetation Index) values of every pixel from all the captured images each year. In the process, we filtered the bad quality pixels like clouds, ice, snow, shadow, etc. A filtering step is added to distinguish the non-vegetation and vegetation pixels first. The overall workflow uses state-of-art remote sensing classification techniques. Machine learning (ML) algorithms like KNN, Gaussian Naïve Bayes, Decision Tree, AdaBoost, Random Forest, SVM, and Neural Networks were used simultaneously to evaluate the approach. The study area is located in the state of Nebraska and the satellite imagery used includes Landsat 8 surface reflectance products. The ground truth data comes from field surveys, roadside samples, and USDA (United States Department of Agriculture) crop maps. Google Earth Engine was used to accelerate the data pre-processing. We tested all the ML models on two sets of experiments: GP and non-GP. In each set, the training has only one-year data (2013) and the testing uses the rest years (2014-2018). The results show that the proposed GP-based approach can significantly improve the classification precision by $\sim 15 \%$ (from $\sim 70 \%$ to $\sim 85 \%$ ) on average. This research proves that greenest pixels have large potential and should be considered as the major input data in the crop mappings in the future.

Keywords: greenest pixel; machine learning; land cover; remote sensing; image classification.

\section{Introduction}

Remote sensing plays a big role in agriculture monitoring today. Satellites, drones, airplanes take thousands of images about farm fields every day (Sun, Di, and Fang 2018; Pinter Jr et al. 2003; Adão et al. 2017; Sun, Di, Sun, et al. 2019; Sun, Di, Fang, et al. 2019). A lot of useful information is hidden in these images. Via these images, people can monitor the recent progress of crops, analyze the impacts of disasters, and study the vegetation phenology (Sun 2019; Zhong et al. 2019). To reveal the contained information and make them easy to read, scientists produce many thematic maps regarding various application purposes. 
For instance, agricultural drought maps are generated to guide the irrigation (Sun, Di, Zhang, Fang, Yu, Lin, Tan, et al. 2017; Sun, Di, Zhang, Fang, Yu, Lin, Tang, et al. 2017; Zhong et al. 2019). Vegetation health maps are derived to guide the use of chemicals to boost the yields and protect the crops from disease, weeds, infestation, etc (Sun et al. 2014; Yang et al. 2011). The crop distribution map is normally produced annually to show the crop types growing on the fields in that year (Xiong et al. 2017). Due to the low cost and high availability of remote sensing images, remote sensing image classification is now the major method to produce in-time and large-scale agricultural thematic maps (Moran, Inoue, and Barnes 1997).

The common classification techniques can be categorized into two types: unsupervised classification and supervised classification (Banman 2002). Thematic classification tasks like crop distribution mapping should use supervised classification (Sun et al. 2016). To assist the supervised classification on a large scale, a tremendous amount of ground truth data points is required to train the models and validate the results. However, the cost of collecting ground truth data is much higher than remote sensing data at present, which is one of the major bottleneck problems in crop mapping. USDA (United States Department of Agriculture) has devoted long-term efforts to survey the crop fields every year and collected a complete systematic set of ground measurements by their field offices. Use that data, USDA NASS (National Agricultural Statistics Service) produces Cropland Data Layer (CDL), annual crop maps of continental United States at the resolution of 30 meters by classifying the imagery of Landsat and some other satellites (Johnson and Mueller 2010; Boryan et al. 2011). See5, a renowned decision tree software, is used to carry out the classification for CDL (Quinlan 1997). CDL owns a very high accuracy that ranges from $82 \%$ to $95 \%$. Some large farm areas could even reach to 97\% (Boryan et al. 2011; Sun, Di, and Fang 2018). Recent new research shows that applying new supervised classification could improve the accuracy further while reducing the dependence on ground truth data by creating reusable classification models. Many papers highlighted ML models could increase the accuracy and reusability significantly (Duro, Franklin, and Dubé 2012; Waldner, Canto, and Defourny 2015; Fritz et al. 2015; Xiong et al. 2017; Gao et al. 2017).
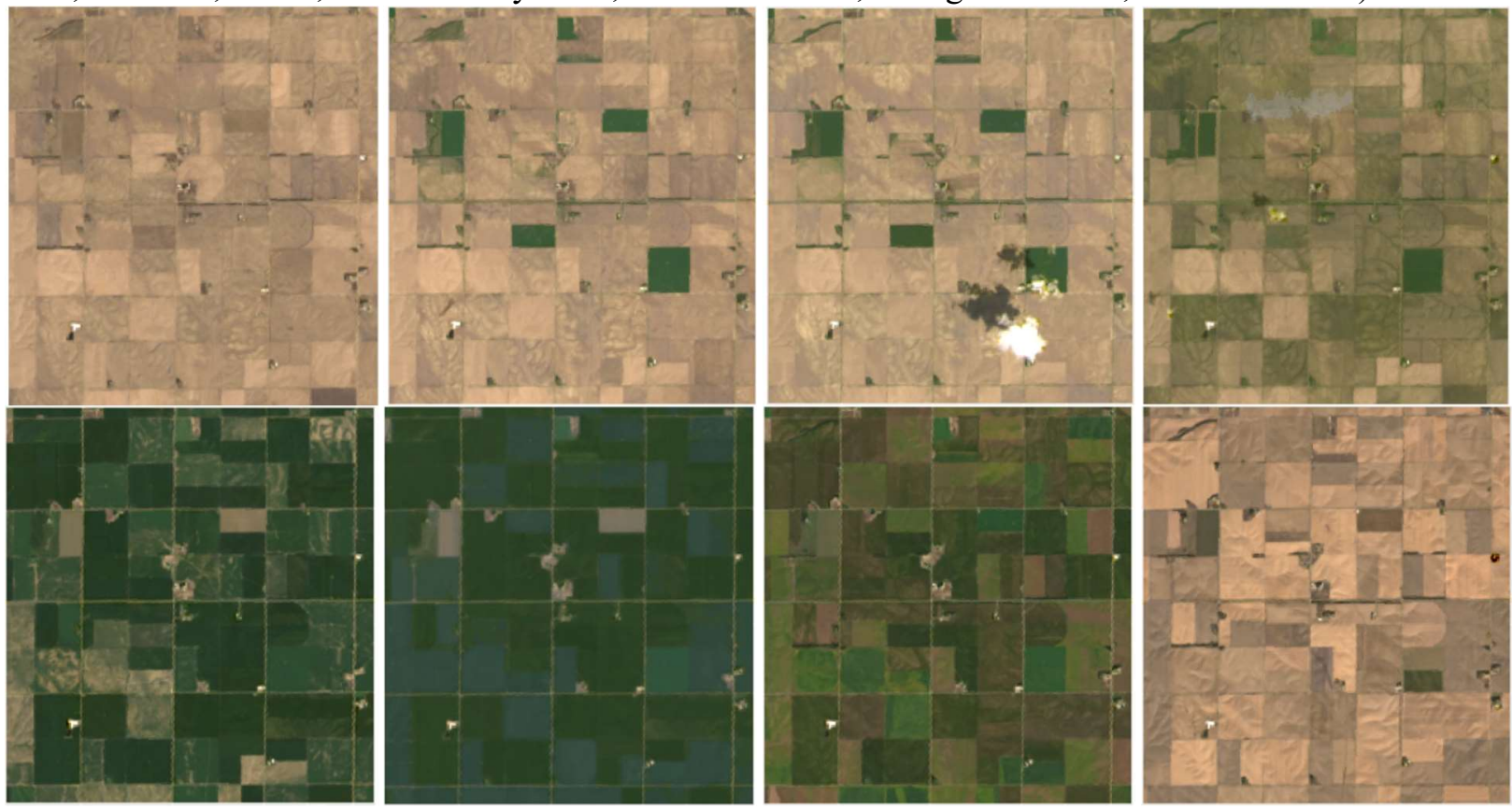

Figure 1. The raw true-color Landsat images (top row from left to right: 2018-04-05, 2018-05-07, 2018-

05-23, 2018-06-08; bottom row from left to right: 2018-07-10, 2018-08-11, 2018-09-12, 2018-11-15)

Besides ground truth data, the selection of remote sensing images is another challenge in improving thematic classification quality. The phenology of various crop types has huge differences, which results in that not all the remote sensing images are suitable for classification. Fig. 1 shows the Landsat true-color 
images (Roy et al. 2014) of the same area on different days in 2018. This area is located at the heart of Nebraska, a major corn/soybean state in the nation (Sun 2019). The image of April 5 has no green fields, indicating that no crops were planted or not emerging yet. This image doesn't contain any information on crops. In the images on May 7 and 23, several alfalfa fields became green as they were planted early and grew fast. In the June 8 image, cornfields started to green up while some of the alfalfa fields had been cut for the first time (alfalfa fields turned light green). The black layer stage is when the corn is fully mature and ready to harvest. For July 10, Corn in Nebraska is at the peak of vegetative grow, at the V16 stage. In the July 10 image, corn in Nebraska typically entered the V16 stage (V: vegetative growth stages; R: reproductive growth stages), while soybeans also are in V stages. On August 11, corn reached the milk (R3) stage and soybean have reached full maturity and are ready for harvesting. Alfalfa was harvested for another time and turns light green again. Based on a report from USDA NASS, soybean and corn are matured and ready to harvest in mid-September to early October. On the image of September 12, most corn fields are at the late reproductive stage, and alfalfa grows up again. In the November 15 image, all the fields returned to idle like the first image of April 5. The first four images and the last two images are not suitable to identify corn and soybeans but good for recognizing alfalfa and field ridges. The revisiting period of the same place of every satellite differs every year and so do the crop phenological stages, which are greatly influenced by the farmer's decisions and weather conditions. Due to these uncertainties, searching and selecting remote sensing images suitable for classification takes a lot of manual efforts and is labor-consumptive to scale up for a large geographic area and users normally need to spend a lot of money to obtain high spatial-resolution crop maps.

To address these challenges, this paper proposes a novel approach using greenest images and machine learning (ML) models in producing crop maps. Greenest images are calculated by extracting the greenest pixels from all the captured images in one year. The greenness is measured by NDVI (normalized difference vegetation index) (EOS 2019). In other words, the pixels with the highest NDVI values are considered as the greenest pixels. The pixels in the greenest image should all be the greenest of the year. Classic ML models are created to take the greenest images as inputs and output a thematic map of crop types. The resulting map is a raster image, in which the pixel values correspond to crop categories. For instance, 1 represents corn, and 5 represents soybean. Fig. 2 shows a comparison between original true-color Landsat images, greenest images, and thematic crop maps in 2018. It is clear that all the crop fields in the greenest image are green and covered by crops, unlike the original Landsat images always including some idle fields. The thematic crop map renders different land cover types with various colors for better readability. In the CDL hierarchy, there are more than 100 crop types.
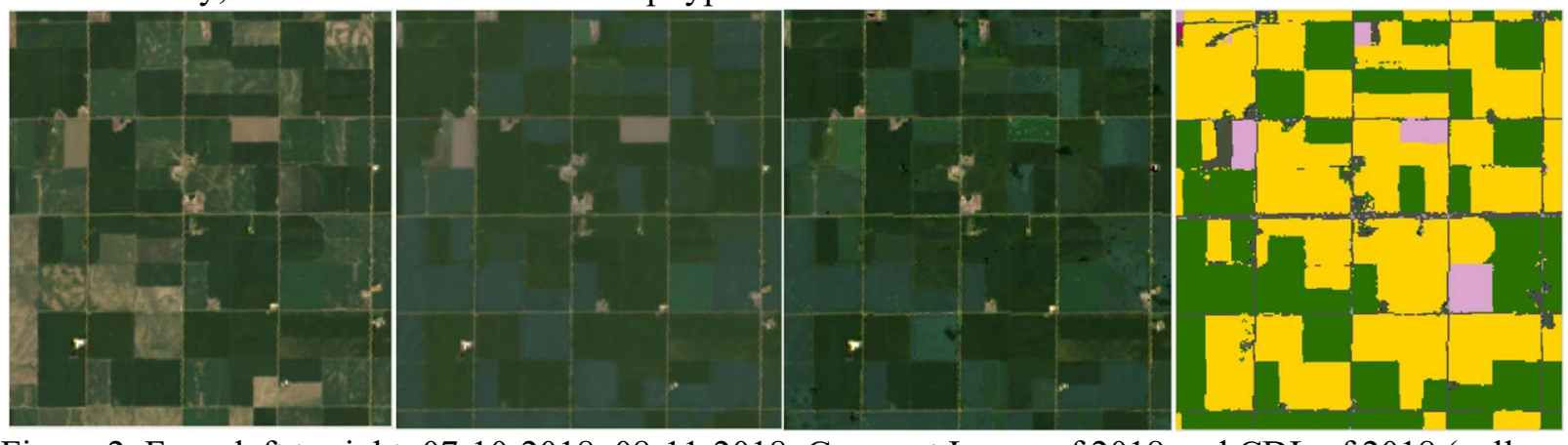

Figure 2. From left to right: 07-10-2018, 08-11-2018, Greenest Image of 2018 and CDL of 2018 (yellow: corn; green: soybean; pink: alfalfa; black: roads)

In this work, a new workflow streamlining the preparation and feeding of greenest images into ML models is built and experimented. The workflow translates the ML model results to crop maps which are ready for stakeholders to use. The whole workflow involves dozens of tools, resources, and libraries, and requires better management to improve efficiency. The study area chooses a farm located in the heart of 
Nebraska and the data sources are Landsat 8. The images are preprocessed to become consumable arrays in ML models. Classic ML models including KNN, Gaussian Naïve Bayes, AdaBoost, Decision Tree, SVM, Random Forest, and Multilayer Perceptron, are all tested to validate the superiority of the GP approach. The ML models extract the high-level features from the images to identify and recognize the crops. Random Forest and Multilayer Perceptron have been proven to be more accurate and generalizable than the other classification models regarding both major and minor crops. The trained models are proven to be reusable on future images to automatically generate crop maps without needs for collecting new ground truth or retraining.

The remainder of this paper is organized as follows. Section 2 gives the background and motivation of this work. Section 3 introduces the study area and the used datasets. In Section 4, deep learning is briefly introduced as the existing state-of-art work. Section 5 describes the proposed GP-based approach. The experiment and results are described in Section 6 and Section 7 evaluates the performance of the approach by comparing it to other existing approaches. Section 8 concludes the work and gives the work items in the future.

\section{Background}

\subsection{The Role of Farm Maps in Decision Making}

Smallholder farmers are sitting on the front line of agricultural decision making. They are farmers that have more than half of farm work done by family members, cooperative members or neighbors. Decision making is central to farm management. Each decision has an impact on the farm and the farm household. Even deciding to do nothing is a decision and has an impact. The more a farmer is aware of the decisionmaking processes that affect farm and household, the more sustainable the farm will be and the more likely it will be profitable and sustainable. Farm decisions are closely tied to decisions made in the household.

The most characteristic aspects of farming operations are the changes that continuously occur, and the lack of knowledge concerning conditions that affect farm businesses. Market prices, the knowledge of smallholder farmers, techniques, weather, health, and governmental arrangements change all the time. Throughout the agriculture history, farmers had to make decisions based only upon observation and recollection. Both sources are not precise, but it was the best data available (Johnson and Haver 1953).

As scientific methods were introduced, farmers could employ research-derived recommendations to improve production efficiency. Digital technologies are changing the routine of farmers in making their daily decisions. The evolution of those capabilities offers significant benefits for farmers and the entire food supply industry ${ }^{1}$. Farmers have long desired to link measures of outcomes and management actions in farming. Advanced technologies like remote sensing and sensor web give farmers the ability to routinely capture and exploit data to get their desired information. The crop distribution map is an important member of the desired data. Farmers need to get an overview of what other farmers are growing and how many acres are covered by the same crop over the entire region. The information would greatly help them make an economic-wise choice on choosing crops to increase their household income.

Other thematic farm maps will identify areas on the farm that are vulnerable to water contamination, and practices that may contribute to water pollution. The base map of the farm should show all farm fields, the farmstead and barnyard area, associated use areas, wetlands, and forests. Another value of a farm map is for future planning or to best utilize their property. A farm map can also identify soil types and other characteristics, such as slope, that can be useful in making decisions like grazing plans and crop rotations. A map can be helpful when selecting compost or manure storage areas. It can also play an important role in emergency planning or responding. ${ }^{2}$

\footnotetext{
${ }^{1} \mathrm{https}: / /$ farmdocdaily.illinois.edu/2020/03/evidence-data-and-farmer-decision-making.html

${ }^{2}$ https://njaes.rutgers.edu/fs1189/
} 


\subsection{Challenges and Opportunities}

Cutting-edge techniques have been utilized in farmland mapping for a long time. Satellite remote sensing is a low cost and reliable way and has been used in the land survey for many years. The remote sensing data is mostly available free and easy to access via web services. Recently, NASA and NOAA have worked with giant tech companies to migrate their big datasets to the commercial clouds that have better scalability and stability for data retrieval. Cloud-native services provide big data processing capabilities that are never seen before, such as Google Earth Engine (GEE). This latest development in satellites and cloud computing has made it convenient to produce agriculture maps in an efficient and economical manner. With the development of intelligent farming devices such as autonomous tractors, remotely monitoring farmland and operating devices to farm efficiently is a big wish of the humankind for the next generation of agriculture. Remote sensing is a key component playing the eye-in-the-sky role in this ambitious vision. It provides critical information about the crops in the field and transfers the information to decision making parties to develop and apply solutions or operations.

\section{Data and Study Area}

\subsection{Study Area}

The study area is within the central region of Nebraska, one of the major corn-belt states. The crops include soybeans, and corn, alfalfa, and some winter wheat. In 2018, the ten leading commodities for cash receipts in Nebraska are cattle and calves, corn, soybeans, hogs, wheat, dairy products (milk), misc. crops, hay, chicken eggs, and potatoes. Among them, corn is Nebraska's most important crop, with much of it going to feed cattle and hogs. The state had 45,900 farms and ranches during 2018 and the average operation consisted of 980 acres (397 ha). The landscape of Nebraska has a 4,584 feet elevation difference, and the average annual precipitation decreases by one inch every 25 miles from east to west. The agricultural industry in Nebraska shows a diverse change from one side to the other.

\subsection{Data Sources}

\section{(1) Landsat 8}

Landsat 8 is the latest in-orbit satellite of the Landsat program and was launched in 2013 (Roy et al. 2014). The satellite has two major sensors onboard: Operational Land Images (OLI) and the Thermal Infrared Sensor (TIRS). It circles the Earth every 99 minutes and has a 16-day repeat cycle for any location on the Earth. It acquires about 740 scenes a day on the Worldwide Reference System-2 (WRS-2) path/row system. The mostly used bands come from OLI include nine bands at 30-meter resolution. The band range is kept consistent with the bands of old TM/ETM sensors carried by previous Landsat satellites.

(2) Cropland Data Layer (CDL)

CDL is a georeferenced raster crop map of the U.S. at a spatial resolution of 30 meters. It is derived by classifying satellite imagery using reliable classification algorithms and the massive amount of ground truth data collected by the United States Department of Agriculture (USDA) National Agricultural Statistics Service (NASS). It is released yearly and normally published in next February. The accuracy in the crop fields ranges from $85 \%$ to $95 \%$. The data is available for the lower 48 states since 2008 . Before that, only a few states are available. Due to its high quality, it has been used in many major researches related to agriculture by both academia and industries.

\section{(3) Roadside Survey}

The ground truth data of USDA, Common Land Unit (CLU), is not in the public domain (USDA 1998). To validate our classification results, we collected ground observations via very high-resolution images, field surveys, and road trips. Many people in the U.S. enjoy identifying and observing farms and farm activity while on a road trip. We made several trips to Nebraska and drive a big circle from Omaha to Ogallala and drive back via Norfolk. The trips occurred in July and we took 1,095 photo samples in total 
showing the growing crops. We processed all the photos and translate them into a collection of ground truth polygons as shown in Fig. 3. This work extracts and utilizes those samples located in the study area.

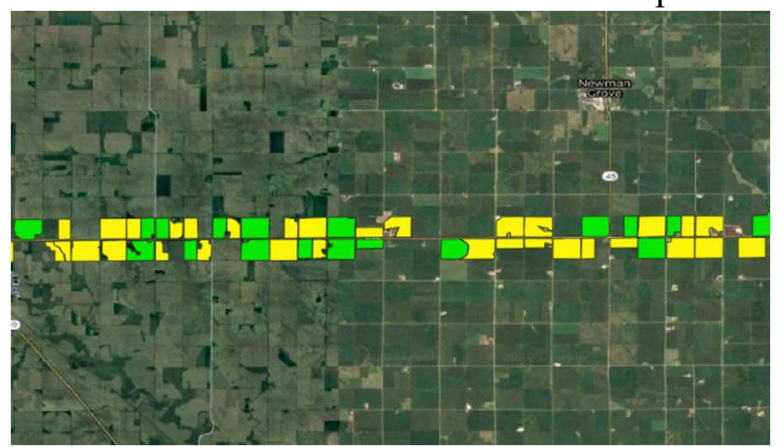

Figure 3. Roadside Survey (yellow: corn; green: soybean)

\section{Greenest Pixel}

NDVI is a measure of the greenness of land surface and associated objects. The values range from 0 to 1. Using greenness is a great way to deal with the discontinuities between Landsat paths, in the median pixel composite due to the differences in phenology as a result of images in adjacent paths being collected at different times. One way to minimize that is to set pixel values in the composite from roughly the same phenological stage, for example, the time of maximum greenest of plants (when the leaves are on and photosynthetically active). The entire time series is examined and the pixel with the maximum value in the NDVI band is set as the composite value. Vegetated areas all appear green because NDVI is highest when the vegetation in the pixel is photosynthetically active. By defining the max greenness by the maximum NDVI, we can use the mosaic method to make a composite in which each pixel contains the maximum NDVI pixel from the collection. The result will be the greenest pixel composite. Fig 2 shows a list of monthly greenest pixel composite images, which are calculated for all the images captured in each month. Indeed, compared to the median composite, the greenest pixel composite is much greener. In the greenest images, there are fewer clouds or bad quality pixels. The images are clean and bright. In the greenest images, the changes in crops over the time series are revealed very explicitly.

The re-composited, cloud-free, greenest Landsat products should be able to provide consistent spatial and temporal comparisons of global vegetation conditions which are used to monitor the Earth's photosynthetic vegetation activity for phonological, change detection, and biophysical interpretations. The composites are calculated from cloud-free and atmospherically corrected gridded surface reflectance. It requires a series of multi-temporal geo-referenced satellite data to be processed into NDVI images. Each pixel will examine its NDVI value and only the highest value is retained for each pixel. After all the pixels have been evaluated, the resulting image is known as the greenest image.
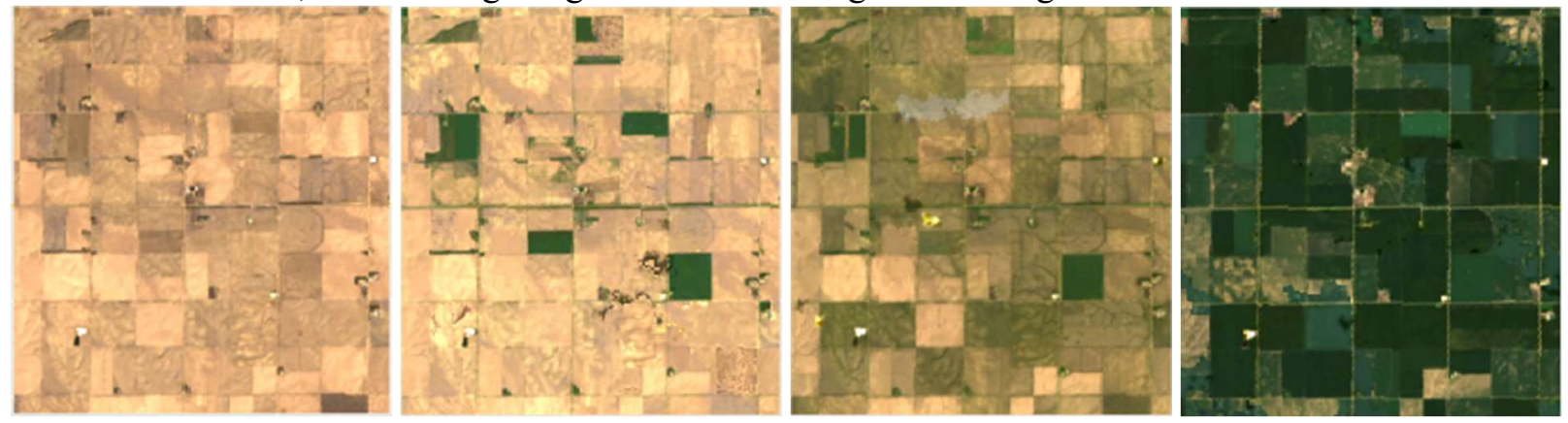

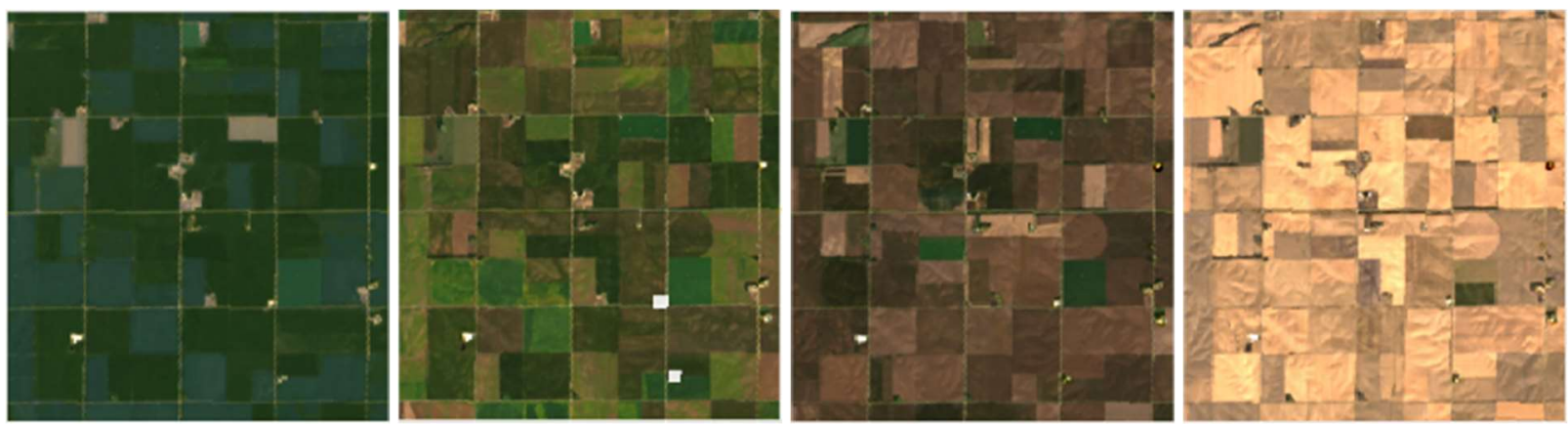

Figure 4. Monthly greenest true-color images in 2018 (top from left to right: April, May, June, July; bottom from left to right: August, September, October, November)

The Landsat time-series dataset could be represented by the following definition:

$$
I=\left\{f_{y, d, \text { lon,lat }, b} \mid y \in[2013,2019], d \in[1,366], \text { lon } \in[-180,180], \text { lat } \in[-90,90], b \in[0,11]\right\}
$$

The set $I$ represents the whole Landsat 8 dataset from 2013 to 2019. The function $f_{y, d, l o n, l a t, b}$ returns observation values. $y$ stands for year, $d$ is the Julian day of the year, lon and lat represent longitude and latitude, and $b$ means the 12 bands from the Landsat Level 1 surface reflectance products. The set of greenest pixels is defined as:

$$
\begin{gathered}
G P=\left\{F_{x, y, b} \mid y \in[2013,2019], b \in[0,11], x \in I, x^{\prime} \in I, Y_{x}=Y_{x^{\prime}}, L^{\prime} O N_{x}=L^{\prime} O N_{x^{\prime}}, L A T_{x}=L A T_{x^{\prime}}, D_{x}\right. \\
\left.\neq D_{x^{\prime}} \text { and NDVI } I_{x}>N D V I_{x^{\prime}}\right\}
\end{gathered}
$$

where the $y$ is the year, $b$ is the bands; $x$ and $x^{\prime}$ are two Landsat pixels; the observation year $Y$, latitude ( $L A T)$ and longitude ( $L O N)$ of $x$ and $x^{\prime}$ are the same; the observation day $D$ of $x$ and $x^{\prime}$ are different; the NDVI of $x$ is greater than the NDVI of any qualified $x^{\prime}$. The NDVI equation is:

$$
N D V I_{x}=\frac{F_{x, 5}-F_{x, 4}}{F_{x, 5}+F_{x, 4}}, x \in I
$$

For Landsat 8, NDVI is equal to the difference between band 5 and band 4 divided by the sum of the two.

\section{Machine Learning Models}

In this section, several classic ML models are introduced. They have been studied for a long time and used in many projects. These models were tested on the GP images for comparison to find out the algorithms with the best performance.

\section{$5.1 \mathrm{KNN}$}

KNN, short for K nearest neighbor, is an entry-level machine learning algorithm (Fox 2010). For every pixel in the imagery, it aims to find the $\mathrm{K}$ samples from the ground measurements which have the most similar features to the pixel. In the K samples, the class with the most votes will claim the pixel.

\subsection{Naïve Bayes}

Naïve Bayes is a family of fast probabilistic algorithms and suitable for processing a large amount of data (Johnson and Mueller 2010). It takes advantage of Bayes' Theorem of probability for prediction of the unknown class under the assumption that the features have strong (naïve) independence among them. It calculates the probability of each class for a given object, and then output the class with the highest probability. Gaussian Naïve Bayes (Gaussian NB) extends the Naïve Bayes to real-valued attributes by assuming a gaussian distribution. First, we can calculate the mean and standard deviation of input values 
(x) for each class to summarize the distribution. In addition to the probabilities for each class, the mean and standard deviations for each input variable of each class must be stored as well. To make predictions, the Gaussian probability density function (PDF) is used. The new input for the variable is plugged into the function, and in return, the gaussian PDF will provide an estimate of the probability of that new input value for that class.

$$
P D F(x, \text { mean }, s d)=\left(\frac{1}{\sqrt{2 \times P I} \times s d}\right) \times e^{-\frac{x-m e a^{2}}{2 \times s d^{2}}}
$$

where $P D F(x$, mean, $s d)$ is the Gaussian PDF, mean and sd are the mean and standard deviation, PI is the numerical constant. We can then plug in the probabilities into the equation above to make predictions with real-valued inputs (Quinlan 1997).

\subsection{AdaBoost}

AdaBoost (USDA 1998) is a classification algorithm by fitting a sequence of weak learners (models that are just slightly better than random guessing) on repeatedly modified versions of the data. The predictions from all of them are then combined through a weighted majority vote to get the final prediction. Each data modification is called a boosting iteration which consists of applying weights $w_{1}, w_{2}, \ldots, w_{n}$ to each of the training samples. Initially, the weights are all set to $1 / N$ ( $\mathrm{N}$ is the total number of samples), so the first step will simply train a weak learner on the original data. In the following steps, the weights will be individually modified, and the learning algorithm is reapplied to the reweighted data. At each step, those incorrectly predicted samples will have their weights increased, whereas the weights are decreased for those correctly predicted. After a few iterations, samples difficult to predict will get higher and higher influence. The classifier will be forced to concentrate on the examples that are missed by the previous iterations in the sequence ${ }^{3}$.

\subsection{Random Forest}

Random forest (Breiman 2001) is an improved algorithm of the basic decision tree. Instead of only one tree, the random forest will create many trees. Instead of just simply averaging the results of the trees (a forest), RF model uses two key ideas to make it random: (1) random sampling of training data points when building trees; (2) random subsets of features considered when splitting nodes. To classify a new object from an input vector, put the input vector down each of the trees in the forest. Each tree gives a classification to vote for one class. The forest chooses the classification having the most votes. When the training set for the current tree is drawn by sampling with replacement, about one-third of the cases are left out of the sample. This OOB (out-of-bag) data is used to get a running unbiased estimate of the classification error as trees are added to the forest. It is also used to get estimates of variable importance. After each tree is built, all of the data are run down the tree, and proximities are computed for each pair of cases. If two cases occupy the same terminal node, their proximity is increased by one. At the end of the run, the proximities are normalized by dividing by the number of trees. Proximities are used in replacing missing data, locating outliers, and producing illuminating low-dimensional views of the data. Because of the out-of-bag estimate internally, there is no need for cross-validation or a separate test set to get an unbiased estimate of the test set error.

Random forest is much better to avoid overfitting than one single decision tree. Overfitting means that the model not only learns the actual relationships in the training data, but also the noises. The noise part will make the trained model yield a very poor performance on new data.

${ }^{3}$ https://scikit-learn.org/stable/modules/ensemble.html\#adaboost 


\subsection{Support Vector Machine (SVM)}

SVM (Cortes and Vapnik 1995) is inspired by the idea to find a hyperplane that best divides a dataset into two classes. Support vectors are the data points nearest to the hyperplane. If some support vectors are removed, the hyperplane will move too. So the support vectors are considered as the critical elements of a data set. The distance between the hyperplane and the nearest data point from either set is known as the margin. The goal is to choose a hyperplane with the greatest possible margin between the hyperplane and any point within the training set, giving a greater chance of new data being classified correctly. In other words, for linearly separable patterns, SVM could create optimal hyperplane to distinguish them. For the patterns that are not linearly separable, SVM uses the Kernel function to transform original data and map them into a new space. In that space, it would be easier to create the hyperplane.

\subsection{Multilayer Perceptron (MLP)}

MLP (SUTER 1990) is one basic type of neural network which has multiple hidden layers (perceptron) beside an input layer to receive the signal and an output layer that makes decisions or predictions about the input. The arbitrary number of hidden layers that are the true computational engine of the MLP. They are trained on a strict set of input-output pairs and learn to model the correlation between the inputs and outputs. Training involves adjusting the parameters, or the weights and biases, of the model to minimize error. Backpropagation is used to make those weigh and bias adjustments relative to the error, and the error itself can be measured in a variety of ways. One usual way is by the root mean squared error.

MLP belongs to the family of feedforward neural networks which have two major constant activities: forward and back. In the forward pass, the signal flow moves from the input layer through the hidden layers to the output layer, and the decision of the output layer is measured against the ground truth labels to calculate the errors. In the backward pass, using backpropagation and the chain rule of calculus, partial derivatives of the error function are back-propagated through the hidden layers. The differentiation produces a gradient along which the parameters can be adjusted to make the result of the next attempts closer to the error minimum. The forward and back processes keep going until the error is lowered under a threshold or the error doesn't change by several consecutive loops.

\section{Methods}

\subsection{General description}

This work proposed an approach to integrate the preprocessing steps of greenest pixels into the established machine learning workflow. The approach was designed to map crops including both food crops and cash crops. The training data for machine learning comes from ground-measured data and refined CDL. For the non-cropland area, the land cover labels of NLCD are assumed correct and reused in this approach. The classification is pixel-based using the multiband values of Landsat 8 as inputs and a label value as outputs. The classification module can switch among the algorithms described in Section 5.

\subsection{GP-ML workflow}

This workflow, named GP-ML, includes four main steps: 1) get greenest pixel products and identify potential crop fields; 2) assign a crop type using the CDL, roadside photos and remote sensing images; 3 ) perform classification using the mentioned machine learning models and the prepared training dataset; and 4) refine the incorrect initial crop types in those edge areas of crop fields, and integrate the CDL product, non-crop land cover, refined ground samples, and the ML generated results to produce the final crop map products. These four steps are further described as follows.

\subsubsection{Identify Potential Crop Fields}

To identify potential crop fields, we first compiled the areas where the Landsat imagery fully covered in the growing season and retrieve all the available images. NDVI is calculated for every Landsat image which filtered clouds and shadows based on the quality band. We extracted the pixels whose NDVI values 
are larger than 0.4 . The threshold is set to be 0.4 based on empirical experiences and numerous experiments by referring to ground photos and samples. The pixels with NDVI larger than 0.4 have a very high chance to be at least $60 \%$ vegetation area. However, it is hard to distinguish the forests from crops simply using NDVI. We used the cultivated mask layer from USDA to further extract the pixels to get the crop fields.

\subsubsection{Image Classification}

The greenest pixels of the recognized crop fields and the corresponding crop types are used as the training dataset for machine learning classification. The combined training data, along with all the Landsat images of 2015 are used as input data into the machine learning classifiers to train the models. The trained model will be used to produce crop maps for the next few years, e.g., 2015, 2016, 2017, 2018. During the model training, the connections between the Landsat observations and the crops growing in the fields will be learned and used to establish the rules (Random Forest, Decision Tree), create hyperplanes (SVM) and tune the weights (MLP). For the other classifiers, the encoding of crop types uses a continuous list of integers each of which represents a land cover type and all the null classes in the CDL hierarchy are cleaned. For the MLP, one-hot encoding is used to encode the crop types into arrays instead of a particular integer. The classification is mainly based on the spectral information of the pixels. Not much shape and texture information are utilized in the process. The experiments on the two sets of experimental data will reveal the benefits of greenest pixels and the better ML models for classification on greenest pixels.

\subsubsection{Accuracy assessment}

Confusion matrix-based metrics are used to evaluate the performance of GP-ML and compare the machine learning models, evaluating its improvements over the non-GP approach. The used metrics include precision, recall, and F-1. The assessment focused on the cropland areas since the goal of the method was to produce crop field maps. Two sets of sample points were selected: the first set was used to evaluate the overall accuracy of the method to map crop fields based on greenest pixels; the second set was used to assess the accuracy of the non-greenest pixels. To draw the first set of sample points, random sampling is used by extracting all the areas which have NDVI values larger than 0.4 . 100 points could provide a reasonably good accuracy estimate. For each pixel, a reference point of crop types was derived by visual interpretation from roadside photos and high-resolution images provided by USDA NAIP (National Aerial Imagery Program).

\subsubsection{Post-refinement Processing}

We refined the results by correcting errors such as the pixels which are classified as crops which contrast to the ground truth and the inappropriate crop labels in the edge areas of the crop fields and the water shallow region. The cultivated layer from USDA may exclude some crop fields or include some noncrop fields. To correct this, we visually identified some missing fields by comparing the crop maps from machine learning models with high-resolution satellite images. After we confirm there is a crop field, we will manually change the mask and rerun the model to generate a crop type for the pixel. For those pixels in wetlands or edge areas, we will determine by the neighbor pixels. If over $60 \%$ of neighbor pixels are the same crops, we will assign the same crop type to the pixel.

\section{Results}

\subsection{Image Processing}

Google Earth Engine is used to collect all the intersected Landsat scenes with the study area and 100 roadside images were processed to create 6 greenest pixel images from 2013 to 2018. The quality of the greenest images is significantly improved and the missing pixels are much less and the cloud and shadow areas in the original Landsat scenes are replaced or filled using the greenest observation. Jupyter notebook is used as the developer environment. Scikit-learn library is used to train machine learning models. The training of each model takes about 2 hours. The SVM and Random Forest takes more time than other models. All the models are trained and tested on the same training dataset. We use the images from 2013 
as the training samples and test on the images from the next five years (as shown in Table 1). Only oneyear data is used for training because we want to shorten the training time cost and emphasize the generality test of the models. All the models are applied to both the greenest pixel and raw pixel training datasets. The pixels are filtered by cloud and vegetation masks before they are fed into the ML models for testing. The predicted results are ensembled back into images corresponding to their input pixel locations.

Table 1. Experiment Settings

\begin{tabular}{|c|c|c|c|}
\hline Experiment & Train & Test & Methods \\
\hline \multirow[t]{7}{*}{ Greenest Pixel } & \multirow{7}{*}{$\begin{array}{l}2013 \\
\text { Yearly Greenest Pixels }\end{array}$} & \multirow[t]{7}{*}{$2014-2018$} & Random Forest \\
\hline & & & SVM \\
\hline & & & Gaussian Naïve Bayes \\
\hline & & & Decision Tree \\
\hline & & & AdaBoost \\
\hline & & & KNN \\
\hline & & & MLP \\
\hline \multirow[t]{7}{*}{ Raw Landsat Pixels } & \multirow{7}{*}{$\begin{array}{l}2013 \\
\text { LC08_L1TP_031031_20130726 } \\
\text { LC08_L1TP_031031_20130827 }\end{array}$} & \multirow[t]{7}{*}{$2014-2018$} & Random Forest \\
\hline & & & SVM \\
\hline & & & Gaussian Naïve Bayes \\
\hline & & & Decision Tree \\
\hline & & & AdaBoost \\
\hline & & & KNN \\
\hline & & & MLP \\
\hline
\end{tabular}

\subsection{Comparison of GP and non-GP}

The results of GP and non-GP in the same year from 2014 to 2018 are compared. In Fig. 5, the top two rows show the non-GP results of Aug 14, 2014, and the bottom two rows show the GP results of 2014. The differences are clear. In general, the GP models outperformed the non-GP models with a very large margin.

For the raw Landsat image, SVM and AdaBoost classified almost all the pixels to cornfields. In the non-GP results of RF and DT, the soybean fields are misclassified to corn, while cornfields are misclassified to soybean fields. Gaussian NB recognized most of the field edges and classify all the pixels into cornfields. KNN generates a similar result to the Random Forest but shows some scattered soybean pixels in the middle of cornfields. MLP result agrees with the Random Forest result on those soybean fields which are wrongly categorized into cornfields. This experiment shows that for raw Landsat images, there is no big improvement by only changing the classification models. The same mistaken pixels can be found in most models' results. Overall, training data is the key to improve the accuracy and generality of the ML models.
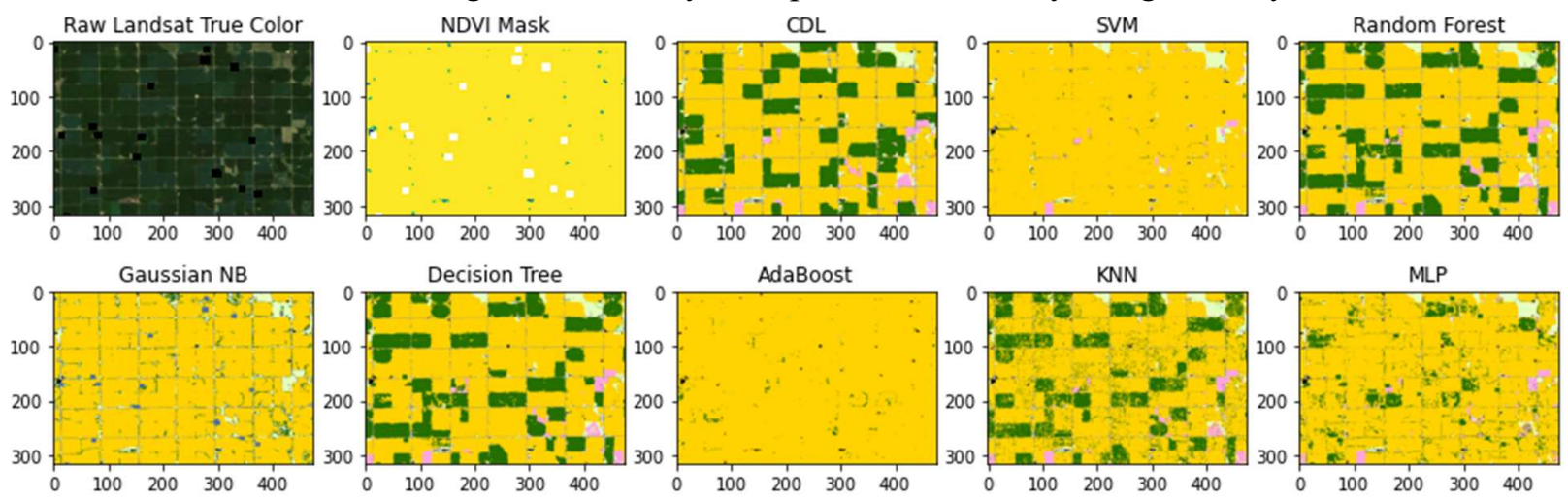

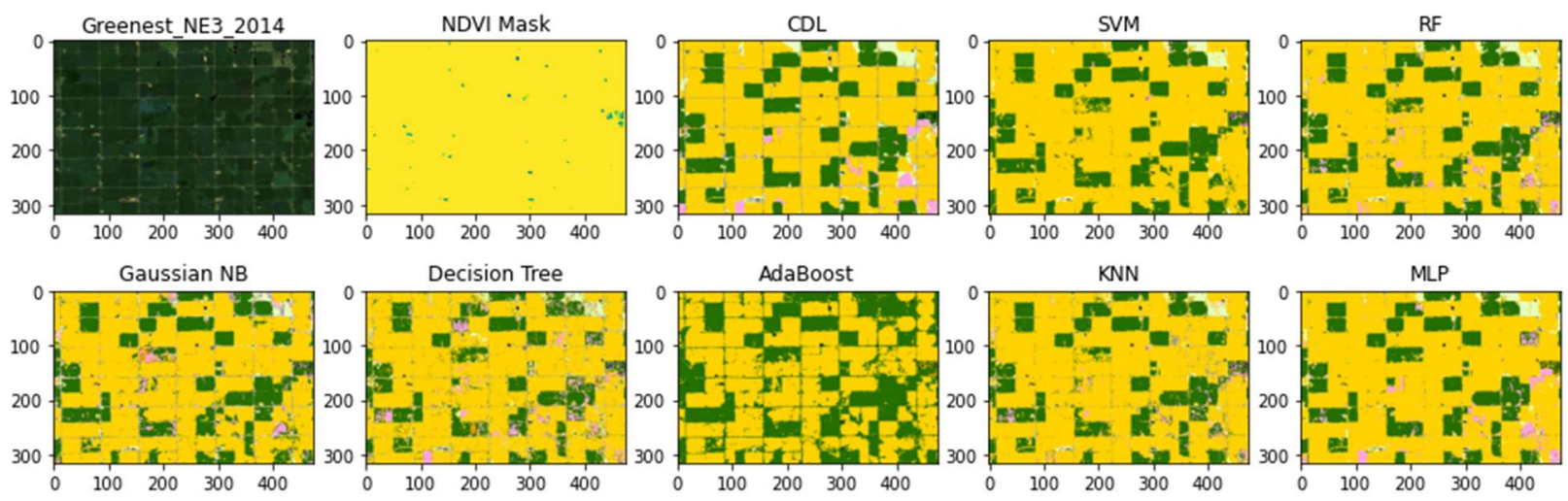

\section{Soybean}

Corn

Grassland ॥ Alfalfa

Figure 5. Comparison between 2014 non-GP $\left(1^{\text {st }}\right.$ and $2^{\text {nd }}$ rows $)$ and $2014 \mathrm{GP}\left(3^{\text {rd }}\right.$ and $4^{\text {th }}$ rows $)$ classification results (top row from left to right: true-color Landsat image, NDVI mask, CDL, SVM, Random Forest; bottom row from left to right: Gaussian NB, Decision Tree, AdaBoost, KNN, MLP)

For the greenest image, all the models generate some reasonably accurate results, with most corn and soybean fields correctly classified. The NDVI mask shows that the greenest pixel image has much fewer blank pixels than the original Landsat image. The grassland regions are greener in the true color images. The alfalfa and grassland are separated very well. The results of SVM, RF, KNN, and MLP agree on the corn and soybean fields. The difference is mainly on some scattered alfalfa pixels. From the perspective of alfalfa, the MLP result has the most identical classification to the CDL. SVM, RF, and KNN all classified some corn pixels into alfalfa. Decision Tree result has noisy texture in the cornfields with soybean pixels all over. Gaussian NB again has the best results on field edges. Gaussian NB did a good judgment, except that one small soybean field in the middle of the image is classified into alfalfa by Gaussian NB. AdaBoost has the poorest prediction in this experiment. It did recognize most of the soybean and cornfields, however, neglect the short-sampled classes like grassland and alfalfa.

After observing the two experiments, it is clear that every ML model did a better prediction on the greenest pixel image than the raw Landsat image. It proves the quality of the training data plays a key role in crop classification. The conclusion could be supported by quantitative metrics of accuracy. The measures of both experiments on the test datasets of four years (2015-2018) are plotted in Fig. 6. Three common indicators are evaluated against the result pixels and the ground truth. The precision of the same models is higher than $76 \%$ on GP but less than 70\% on non-GP in 2015. In the 2015 GP experiment, RF has the most consistent accuracy with the second-highest precision (only after MLP) and the highest recall and F1 score (88\%). In the non-GP experiment on the Landsat image of July 16, 2015, Gaussian NB is the most accurate model on all three metrics. In the 2016 results, the GP-based RF achieves as high as $92 \%$ in precision and $94 \%$ in recall. KNN, MLP, and SVM also did very well on precision and recall. Decision and AdaBoost are not as good as the other four. However, on the non-GP, SVM performs the best with $72 \%$ precision, $75 \%$ recall, and $67 \% \mathrm{~F} 1$. KNN has the lowest precision and F1 and Decision Tree has the lowest recall. In the 2017 result, GP-trained SVM outperforms the other methods with a very consistent value (89\%) on the three indicators. The decision tree is the model with the worst result. For the non-GP in 2017, AdaBoost has the best performance with $74 \%$ precision, $79 \%$ recall, and $74 \% \mathrm{~F} 1$, and $\mathrm{KNN}$ has the poorest measures. In the 2018 GP result, RF again claims the first place with 90\% precision, $90 \% \mathrm{~F} 1$ and $93 \%$ recall. MLP and SVM did the same best. DT and AdaBoost are the two models with the worse predictions. In the nonGP 2018 result, Gaussian NB is the best model and the AdaBoost is the worst.

The experiment results show that the models have very unstable performances on the non-GP data. It is found to be very inconsistent over the four years on the best and worst models. In every year, the nonGP-trained models perform very poorly (50\% 75\% in precision) on new data (trained on 2013 and tested 
on the subsequent years), which reflects the generality of the trained models is bad. However, the same models trained on GP have very solid and relatively steady accuracy (about 80\% 95\%). The model performs better on GP than non-GP in every case. The results have the most consistent precision and recall. The two metrics differ dramatically in the non-GP results, which indicates that the non-GP-trained models either underfit on the majority or overfit on the minority. In conclusion, the GP training dataset could significantly improve the accuracy of the result crop maps on both major crops and minor crops. The models trained on GP have much better generality and accuracy than those trained on non-GP.
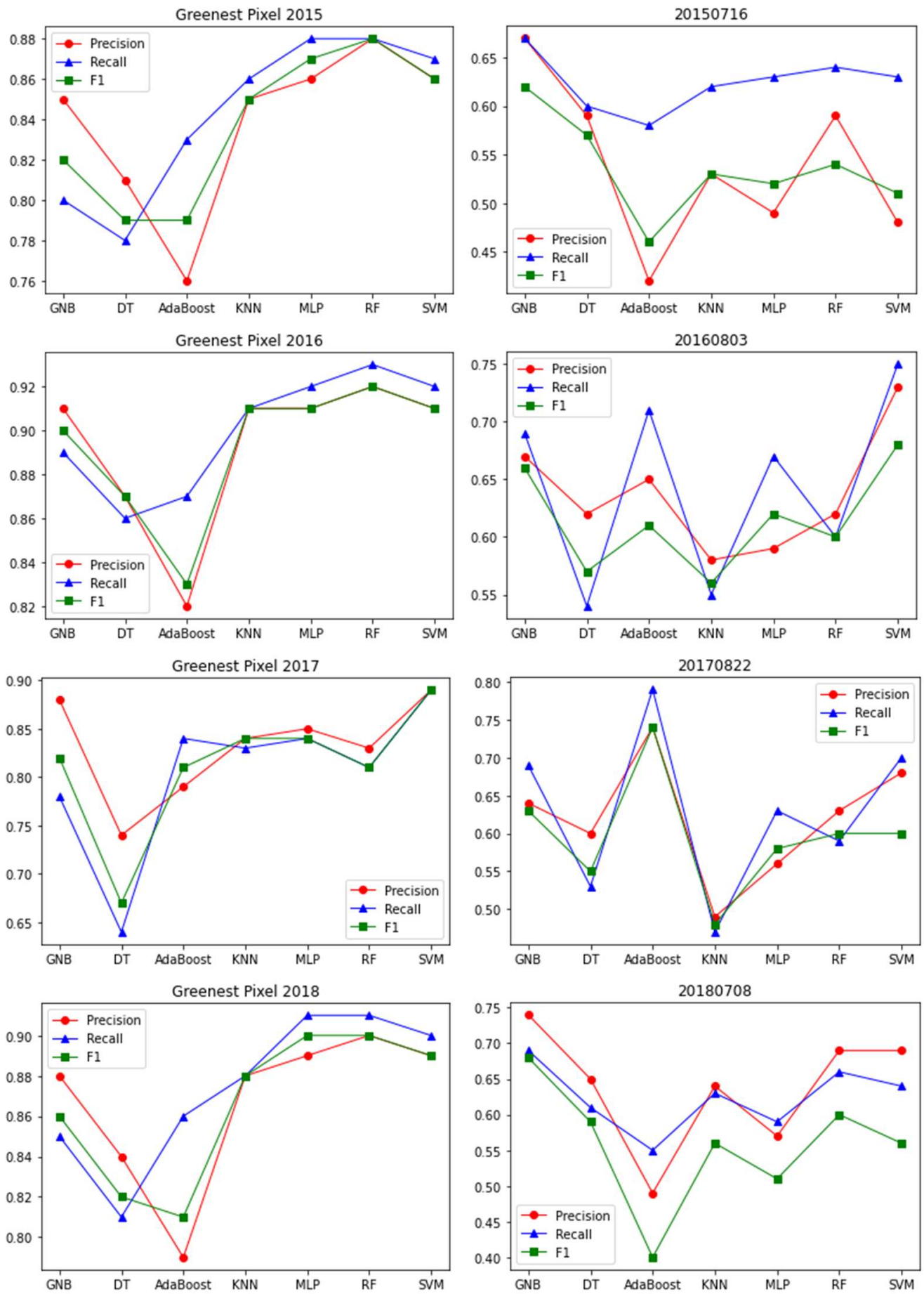

Figure 6. Accuracy Comparison of ML models on greenest pixels (left) and raw Landsat pixels (right) 


\subsection{Comparison of ML Algorithms}

To find the best algorithm for GP, the results of the GP-trained models in all four years (as shown in Fig. 7) are thoroughly studied. Every year, the ML models display a similar pattern in their judgments. SVM can accurately identify soybeans, corns, and grassland, but cannot recognize alfalfa and field edges. SVM misclassified most alfalfa pixels into soybeans. Gaussian NB makes big mistakes in some big soybean fields by classifying them into alfalfa fields. Corn and grasslands are correct. The results of single Decision Tree are very poor with vague and dirty texture and many pixels with classes that don't exist in the region. Single Decision Tree is not recommended for this purpose. AdaBoost did a good job of outlining the field borders. It made the right judgment on the major crops but completely ignored the minor crops. The pixels of grassland and alfalfa are assigned to either corn or soybeans (mostly). KNN outputs a very similar result to the RF and MLP, except for some error spots of soybeans in the middle of cornfields. The KNN result is less smooth and consistent compared to RF and MLP.

The results of RF and MLP are better than other models. models accurately recognize the soybean, corn and grassland fields. It is hard to tell which one of the two is the best. RF delineates the field borders more clearly than MLP while MLP gives better prediction on alfalfa than RF. In other words, if the borders are important target information, RF is recommended; if minor crops like alfalfa are required information, MLP would be the better choice.
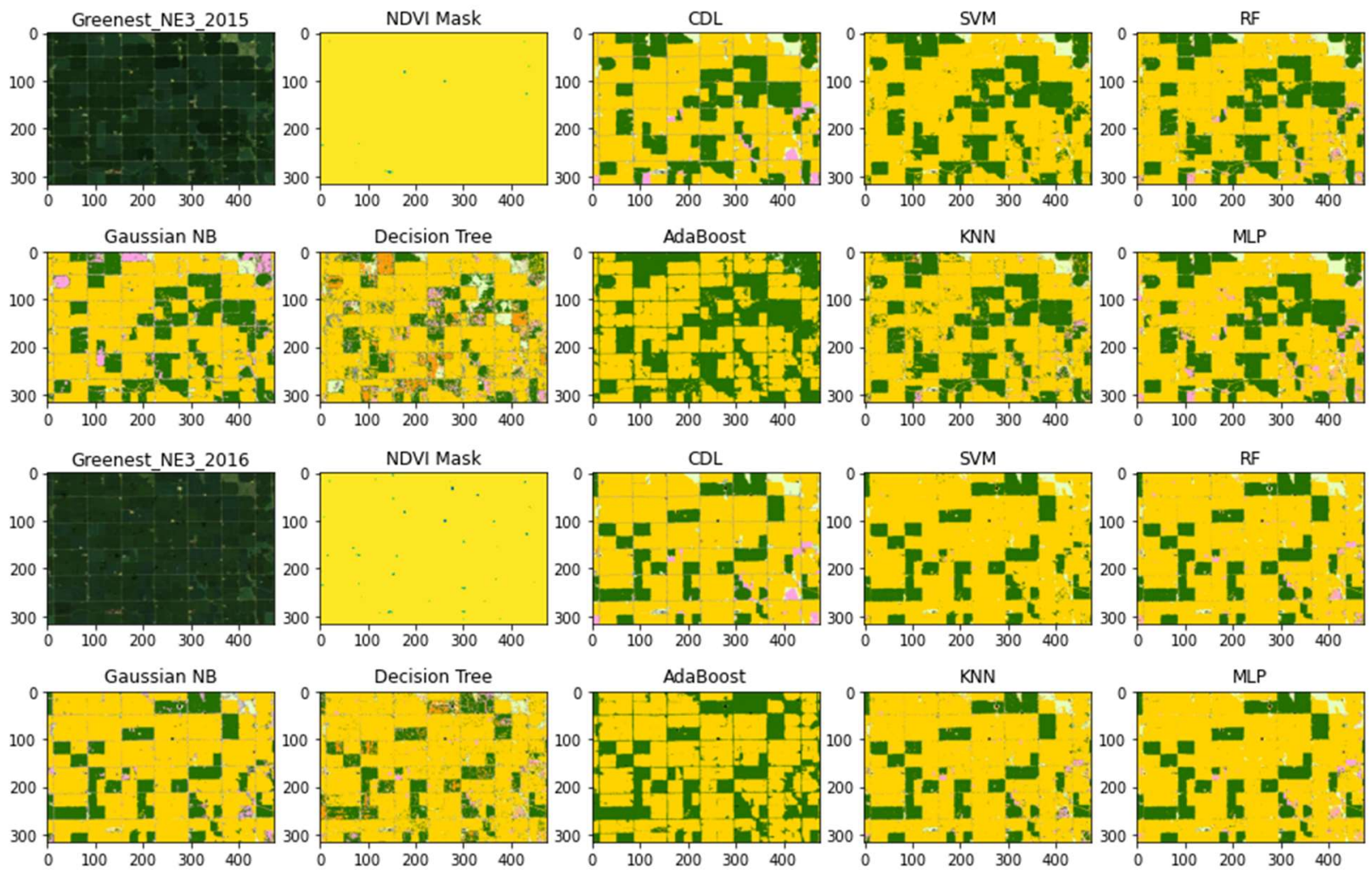

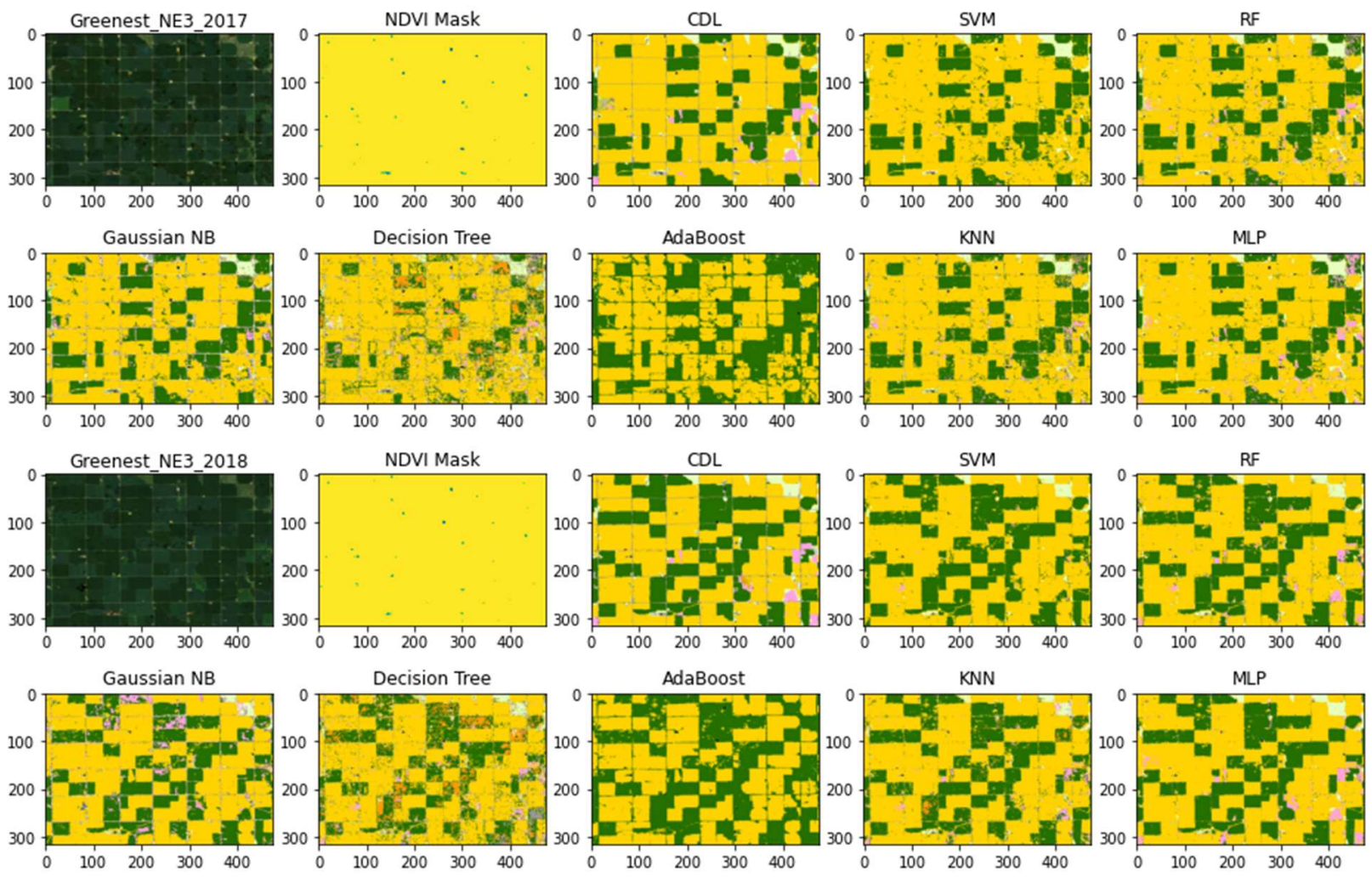

Soybean

\section{Corn Grassland 1 Alfalfa}

Figure 7. Comparison of the Algorithms on greenest pixels (trained on 2015, tested on 2013, 2014, 2016, 2017, 2018)

\section{Discussion}

8.1 Reliability: will the GP-trained models always beat the non-GP models?

Based on the experiment results, the GP-trained ML models outperformed the non-GP-trained models. To rule out the concerns on coincidences, we did another test in the region shown in Fig. 1 and got the same results (GP better than non-GP). The reason is that in the GP the crops and non-crop vegetation are all in their greenest phases, which narrows down the characteristics of the crops into a limited feature space. Therefore, using the GP training dataset the ML models don't need to make wild guesses on which phase the crops are in and require fewer rules or features to distinguish the crops. In other words, the GP training dataset makes the job of ML models much easier than the non-GP training dataset. The superiority of GPtrained models over non-GP models is not coincidental and should be reproducible all the time.

8.2 Generality: will the best ML models for this region always be the top choice for any other regions?

The comparison of ML models in image classification has been studied for a very long time. As one of the common techniques for improvements, hyperparameter tuning could greatly alter the performance of the ML models and bring a lot of uncertainties to the comparison. In this study, we didn't do much tuning on the hyperparameters. All the parameters use their default values provided by the ML library. If the hyperparameter tuning is taken into consideration, the answer to the generality question would be a NO. However, if taking hyperparameter tuning off the table, the answer would be certainly a YES. Without tuning parameters, the Random Forest and Multilayer Perceptron will produce better results for all the regions out of the six listed ML models.

8.3 Scalability: will the same quality results be achieved if the region is extended to a larger scale? 
This question needs more experiments on bigger datasets to answer. At this point, we could only anticipate from the theoretic perspective. The quality of training data would still be key to scale the trained models to be applied in a larger scope. If the samples used for training are less biased and cover most of the unique statuses of the objects, the trained model would obtain more capability to deal with the upcoming new pixels from new images of various regions. The bias of training data is not only about the regions, but also in the classes. Naturally, some crops have more samples (e.g., corn and soybean) while others have fewer occurrences (e.g., lentils). Each class should have a certain minimum number of samples in the training data to ensure the minority crops could be distinguished. In the GP approach, the variety of regions have relatively fewer impacts on the results as the same crop of different regions has very similar spectral characteristics during its greenest stage (assuming the climate of those regions is similar). Overall, better scalability will be achieved with better training data.

\section{Conclusion}

This paper proposes a novel approach using greenest images and ML algorithms in producing crop maps. Greenest images are calculated by extracting the greenest pixel value from all the captured images in one year. The greenness is measured by NDVI (normalized difference vegetation index). The pixels with the highest NDVI values are considered as the greenest pixels. The pixels in the greenest image should all be the greenest. An approach is created to generated and take the greenest images as inputs and output a semantic map of crop types. The map is a raster image, in which the pixel values correspond to crop categories. A new workflow streamlining the preparation and feeding of greenest images into ML models is built and experimented. The workflow will translate the ML model results in crop maps which are ready for stakeholders to use. The whole workflow involves dozens of tools, resources, and libraries, and requires better management to improve efficiency. The study area chooses a farm located in the heart of Nebraska and the data sources are Landsat and USDA NASS CDL. The images are preprocessed to become consumable arrays in ML models. The training among the hidden layers of ML models will extract the high-level features to identify and recognize the crops. RF and MLP have been proven to be more accurate and robust than the other classification models. The trained model is supposed to be reusable on future images to automatically generate crop maps without needs for collecting new ground truth or retraining. The results show that the GP-trained ML models outperformed the non-GP-trained ML models with a very large margin.

In the future, more experiments on a bigger training dataset will be conducted to verify the superiority of the GP-based approach in larger regions. More advanced ML models such as convolutional neural networks and recurrent neural networks will be tested against GP to further improve the accuracy. The readiness of the GP-trained model to produce official crop maps from remote sensing images will be evaluated and carried out in Nebraska in our next step of research.

\section{Reference}

Adão, Telmo, Jonáš Hruška, Luís Pádua, José Bessa, Emanuel Peres, Raul Morais, and Joaquim Sousa. 2017. "Hyperspectral imaging: A review on UAV-based sensors, data processing and applications for agriculture and forestry." Remote Sensing 9 (11):1110.

Banman, Chris. 2014. "Supervised and Unsupervised Land Use Classification." Accessed 4.12.2020. http://academic.emporia.edu/aberjame/student/banman5/perry3.html. 
Boryan, Claire, Zhengwei Yang, Rick Mueller, and Mike Craig. 2011. "Monitoring US agriculture: the US department of agriculture, national agricultural statistics service, cropland data layer program." Geocarto International 26 (5):341-58.

Breiman, Leo. 2001. "Random forests." Machine learning 45 (1):5-32.

Cortes, Corinna, and Vladimir Vapnik. 1995. "Support-vector networks." Machine learning 20 (3):27397.

Duro, Dennis C., Steven E. Franklin, and Monique G. Dubé. 2012. "A comparison of pixel-based and object-based image analysis with selected machine learning algorithms for the classification of agricultural landscapes using SPOT-5 HRG imagery." Remote Sensing of Environment 118:25972. doi: http://dx.doi.org/10.1016/j.rse.2011.11.020.

EOS. "NDVI FAQ: All You Need to Know About NDVI." Accessed 2020.03.16. https://eos.com/blog/ndvifaq-all-you-need-to-know-about-ndvi/.

Fox, N. 2010. "QA4EO guide: QA4EO-QAEO-GEN-DQK-001: a guide to establish a quality indicator on a satellite sensor derived data product (version 4.0)." See http://qa4eo. org/docs/QA4EO-QAEOGEN-DQK-001_v4. 0. pdf.

Fritz, Steffen, Linda See, Ian McCallum, Liangzhi You, Andriy Bun, Elena Moltchanova, Martina Duerauer, Fransizka Albrecht, Christian Schill, and Christoph Perger. 2015. "Mapping global cropland and field size." Global change biology 21 (5):1980-92.

Gao, Feng, Martha C Anderson, Xiaoyang Zhang, Zhengwei Yang, Joseph G Alfieri, William P Kustas, Rick Mueller, David M Johnson, and John H Prueger. 2017. "Toward mapping crop progress at field scales through fusion of Landsat and MODIS imagery." Remote Sensing of Environment 188:925.

Johnson, David M, and Richard Mueller. 2010. "The 2009 cropland data layer." Photogramm. Eng. Remote Sens 76 (11):1201-5.

Johnson, Glenn Leroy, and Cecil Berdeen Haver. 1953. "Decision-making principles in farm management."

Moran, M Susan, Yoshio Inoue, and EM Barnes. 1997. "Opportunities and limitations for image-based remote sensing in precision crop management." Remote Sensing of Environment 61 (3):319-46.

Pinter Jr, Paul J, Jerry L Hatfield, James S Schepers, Edward M Barnes, M Susan Moran, Craig ST Daughtry, and Dan R Upchurch. 2003. "Remote sensing for crop management." Photogrammetric Engineering \& Remote Sensing 69 (6):647-64.

Quinlan, J Ross. 1997. "See5." URL: http: www. rulequest. com/see5-info. html.

Roy, David P, MA Wulder, TR Loveland, CE Woodcock, RG Allen, MC Anderson, D Helder, JR Irons, DM Johnson, and R Kennedy. 2014. "Landsat-8: Science and product vision for terrestrial global change research." Remote Sensing of Environment 145:154-72.

Sun, Jie, Liping Di, Ziheng Sun, Yonglin Shen, and Zulong Lai. 2019. "County-Level Soybean Yield Prediction Using Deep CNN-LSTM Model." Sensors 19 (20):4363.

Sun, Z., C. Peng, M. Deng, A. Chen, P. Yue, H. Fang, and L. Di. 2014. "Automation of Customized and Near-Real-Time Vegetation Condition Index Generation Through Cyberinfrastructure-Based Geoprocessing Workflows." Selected Topics in Applied Earth Observations and Remote Sensing, IEEE Journal of 7 (11):4512-22. doi: 10.1109/jstars.2014.2377248.

Sun, Ziheng. "Automatically Recognize Crops from Landsat by U-Net, Keras and Tensorflow." Accessed 1/26/2020. https://medium.com/artificial-intelligence-in-geoscience/automatically-recognizecrops-from-landsat-by-u-net-keras-and-tensorflow-7c5f4f666231.

Sun, Ziheng, Liping Di, and Hui Fang. 2018. "Using long short-term memory recurrent neural network in land cover classification on Landsat and Cropland data layer time series." International Journal of Remote Sensing 40 (2):593-614. 
Sun, Ziheng, Liping Di, Hui Fang, Annie Bryant Burgess, and Navya Singh. 2019. Deep Learning Cyberinfrastructure for Crop Semantic Segmentation. Paper presented at the AGU Fall Meeting 2019.

Sun, Ziheng, Liping Di, Chen Zhang, Hui Fang, Eugene Yu, Li Lin, Xicheng Tan, Liying Guo, Zhongxin Chen, and Peng Yue. 2017. Establish cyberinfrastructure to facilitate agricultural drought monitoring. Paper presented at the Agro-Geoinformatics, 2017 6th International Conference on.

Sun, Ziheng, Liping Di, Chen Zhang, Hui Fang, Eugene Yu, Li Lin, Junmei Tang, Xicheng Tan, Ziao Liu, and Lili Jiang. 2017. Building robust geospatial web services for agricultural information extraction and sharing. Paper presented at the Agro-Geoinformatics, 2017 6th International Conference on.

Sun, Ziheng, Hui Fang, Liping Di, Peng Yue, Xicheng Tan, and Yuqi Bai. 2016. "Developing a web-based system for supervised classification of remote sensing images." Geolnformatica:1-21.

SUTER, BRUCE W. 1990. "The multilayer perceptron as an approximation to a Bayes optimal discriminant function." IEEE Transactions on Neural Networks 1 (4):291.

USDA, FSA. 1998. "Common land unit." FSA Handbook, 8-CM.

Waldner, François, Guadalupe Sepulcre Canto, and Pierre Defourny. 2015. "Automated annual cropland mapping using knowledge-based temporal features." ISPRS Journal of Photogrammetry and Remote Sensing 110:1-13.

Xiong, Jun, Prasad S Thenkabail, Murali K Gumma, Pardhasaradhi Teluguntla, Justin Poehnelt, Russell G Congalton, Kamini Yadav, and David Thau. 2017. "Automated cropland mapping of continental Africa using Google Earth Engine cloud computing." ISPRS Journal of Photogrammetry and Remote Sensing 126:225-44.

Yang, Zhengwei, Liping Di, Genong Yu, and Zeqiang Chen. 2011. Vegetation condition indices for crop vegetation condition monitoring. Paper presented at the Geoscience and Remote Sensing Symposium (IGARSS), 2011 IEEE International.

Zhong, Shaobo, Zhanya Xu, Ziheng Sun, Eugene Yu, Liying Guo, and Liping Di. 2019. Global vegetative drought trend and variability analysis from long-term remotely sensed data. Paper presented at the 2019 8th International Conference on Agro-Geoinformatics (Agro-Geoinformatics). 